\title{
Need for increased clinical awareness of tuberculosis during pregnancy and puerperium in India
}

\author{
Kavita P. Jain, Pratishtha B. Chaudhari*
}

Medical Officer, UHP Airoli, NMMC Health Department, New Mumbai 400610

Received: 03 October 2016

Revised: 01 November 2016

Accepted: 02 November 2016

\section{* Correspondence:}

Dr. Pratishtha B. Chaudhari,

E-mail: pratishthabanga@gmail.com

Copyright: () the author(s), publisher and licensee Medip Academy. This is an open-access article distributed under the terms of the Creative Commons Attribution Non-Commercial License, which permits unrestricted non-commercial use, distribution, and reproduction in any medium, provided the original work is properly cited.

\begin{abstract}
TB is leading cause of morbidity and mortality in women of reproductive age in India. TB is under-diagnosed among women due to a number of barriers to care. The timely diagnosis of tuberculosis (TB) during pregnancy is of utmost importance to both the mother and the fetus. Strategic efforts to identify and document active Tb disease in pregnant and post-partum cases are key steps to successful treatment and program execution. To stay on track with the Sustainable Development Goal 2030 by WHO to end Tb by 2030 and reduce tuberculosis-related morbidity and mortality, more concentrated efforts on tuberculosis in pregnant and postpartum women is critical and innovative approaches to case detection are needed. Intensified research and innovation are the important pillars and components of sustainable Development Goals 2030 and discovery, development and rapid uptake of new tools, interventions and strategies are called for. This systematic review aimed to gather and evaluate evidence based studies on TB diagnosis in pregnancy, as per WHO standard guidelines for TB care - RNTCP, in order to recommend proposals for better practices to improve active TB diagnosis for pregnant women, aligned with the End Tb Strategy of SDG2030. The data search for studies on TB care in pregnancy the authors intended to select those addressing screening/diagnosis, prevention of mother-to-child transmission for women in pregnancy. The authors put forth evidence that there should be special provision made for screening, timely diagnosis and management of pregnant women with TB.
\end{abstract}

Keywords: Early case detection, Mother and child health, Pregnancy, Postpartum, Tb goals, Tuberculosis

\section{INTRODUCTION}

According to the WHO recommendations for microbiological screening of $\mathrm{TB}$ in pregnancy for high HIV and TB-burden antenatal clinics, National TB Programmes (NTPs) should make a concerted effort to capture pregnancy-associated TB and to follow-up on perinatal outcomes. Simple efforts like addition of entry column for 'pregnancy status' in RNTCP patient card, 'cough of any duration' in a pregnant patient to be recorded and examined for $\mathrm{Tb}$, to maintain a high degree of suspicion and close follow up for any signs and symptoms of $\mathrm{Tb}$, during pregnancy and post-partum period - such efforts utilise the existing resources while increase the case detection rates of $\mathrm{Tb}$ in pregnant patients in India.
The tuberculosis pregnancy interaction is like a doublededged sword, one edge being the impact of tuberculosis on pregnancy and new-born, while the other edge is effect of pregnancy on progression of tuberculosis. The impact on pregnancy is variably reported in literature - to improve, worsen, or have no effect on TB. Both pregnancy and TB can adversely affect each other and lead to poor outcomes. In either case, the timely diagnosis of tuberculosis (TB) during pregnancy is of utmost importance to both the mother and the fetus since untreated disease carries much greater risk to both. Heightened awareness of the risk of TB in pregnant patients is the need of the hour. Strategic efforts to identify and document active $\mathrm{Tb}$ disease in pregnant and post-partum cases are key steps to successful treatment and program execution. 
This systematic review aimed to gather and evaluate evidence based studies on TB diagnosis in pregnancy, as per WHO standard guidelines for TB care, RNTCP, in order to recommend proposals for better practices to improve active TB diagnosis for pregnant women, aligned with the End Tb Strategy. The data search for studies on TB care in pregnancy the authors intended to select those addressing screening/diagnosis, prevention of mother-to-child transmission for women in pregnancy. The authors try to gather evidence that there should be special provision made for screening, timely diagnosis and management of pregnant women with TB. ${ }^{1}$

\section{Research question}

TB case detection rates are far below the WHO target of $70 \%$ of sputum smear positive cases. TB is leading cause of morbidity and mortality in women of reproductive age in India. ${ }^{2}$ TB is also a major cause of perinatal morbidity and mortality. Maternal co-infection with TB and HIV increases the risk for perinatal transmission of HIV. ${ }^{3,4} \mathrm{~TB}$ is under-diagnosed among women due to a number of barriers to care..$^{5-7}$

To stay on track with the Sustainable Development Goal 2030 by WHO to end $\mathrm{Tb}$ by 2030 and reduce tuberculosis-related morbidity and mortality, more concentrated efforts on tuberculosis in pregnant and postpartum women is critical and innovative approaches to case detection are needed. Intensified research and innovation are the important pillars and components of Sustainable Development Goals 2030 and discovery, development and rapid uptake of new tools, interventions and strategies are called for.

Our research question is are we doing enough to actively identify maximum cases of perinatal $\mathrm{Tb}$ and what efforts can be taken by which we can further upscale the $\mathrm{Tb}$ diagnosis rates in this vulnerable population.

The research questions are:

1. To understand the various presentations and identify those at risk of infection or of developing the disease in pregnancy.

2. To understand the issues of $\mathrm{Tb}$ infection underdiagnosis and control and the multidisciplinary approach to care.

\section{EPIDEMIOLOGY}

About one-third of the world's population (estimated to be about 1.75 billion) is infected with the tubercule bacillus. $^{8}$ The highest probability of having tuberculosis is during the childbearing years of 15 to 49 , with $80 \%$ of all deaths from TB occurring in this group. It is also a significant contributor to maternal mortality, with the disease being among the three leading causes of death among women aged 15-45 years. ${ }^{9}$
Worldwide, TB is the number one infectious cause of death among women, responsible for more than 1 million deaths amongst women each year. TB currently is responsible for more deaths annually than all other causes of maternal morbidity combined. In communities in which TB is endemic, pregnant women are at high risk, especially for infection with resistant organisms. ${ }^{10}$

Tuberculosis not only accounts for a significant proportion of the global burden of disease, In highburden countries, rates of between $0.07 \%$ and $0.5 \%$ were found among HIV-negative women, and between $0.7 \%$ and $11 \%$ among HIV-positive women. ${ }^{11}$ Since many women in high burden countries do not have access to healthcare, the prevalence of $\mathrm{Tb}$ may be underestimated.

TB rates in pregnant women are expected to be higher because of the different demographic composition of this group $^{12}$ and the fact that women from high-prevalence areas often have a higher total period fertility rate. ${ }^{13}$ Two local studies from large London teaching hospitals provided estimated incidence in pregnant women to be 3 and 5 times higher than the respective local background rate (153 to 252 per 100,000 maternities). ${ }^{14}$ In a recent epidemiological modelling study, Sugarman et al. estimated that there may have been $216500 \quad(95 \%$ uncertainty range $192000-247000)$ active TB cases among pregnant women globally in 2011, with the highest case burden ( $41.3 \%$ of cases) in the WHO African region.

The WHO Tuberculosis Report of 2014 stated that in 2013 there were an estimated 3.3 million cases among women, with 510000 deaths; one third of these women were co-infected with HIV. ${ }^{15}$ The report does not mention the word 'pregnancy' or 'pregnant', which is indicative of the fact that most countries do not screen routinely for TB in pregnancy nor do they report the pregnancy status of female TB cases. ${ }^{16}$ The symptoms of TB overlap considerably with those of pregnancy, due to which one South African study has shown that the sensitivity of clinical screening for TB among pregnant women is as low as $28 \%$. This shows that without active lookout and heightened awareness, new case finding programmes among pregnant women, we can never hope to reach sufficient numbers of cases ${ }^{17}$ diagnosed and treated.

There is currently insufficient evidence on the epidemiology of TB in pregnancy globally, and no data on association of pregnancy and Tb. The U.K. General Practice Research Database (GPRD) provided a suitable cohort to investigate the epidemiology of TB in pregnancy in the United Kingdom and to determine whether pregnancy is an independent risk factor for TB to inform future public health and screening policy. Few other national programs collect or report pregnancyspecific tuberculosis data to the World Health Organization (WHO) and it is our recommendation that we include $\mathrm{Tb}$ screening and follow up until puerperium 
as a protocol in RNTCP for a high endemic country like India.

As per the WHO recommendations for microbiological screening of TB in pregnancy for high HIV and TBburden antenatal clinics, National TB Programmes (NTPs) should make a concerted effort to capture pregnancy-associated TB and to follow-up on perinatal outcomes. Collected data should then be analysed and published to better inform guidelines for TB diagnosis and treatment in pregnancy. This is of particular importance with respect to EPTB and MDR-TB. Such cases should be reported to the NTP with immediate effect and efforts made to follow-up these cases, providing the best available care and also recording outcomes.

\section{OUR PROPOSAL}

In the Tuberculosis patient card for RNTCP, a simple addition of entry column for 'pregnancy status' of the female can be made. This shall enhance detailed case recording and will be of immense value for reinforcing the critical Tb-pregnancy interaction in mind of Health Care Provider, will help future analysis and hypothesis generation.

Pregnant - yes no not applicable

Post-partum (until 6 weeks) - yes no not applicable

\section{Gaps in knowledge and practice}

The major problem concerning TB diagnosis for pregnant women is the delay in diagnosis. Delay in diagnosis is defined as the duration from symptom onset to confirmation of diagnosis. It may range from 7 days to 6 months. Bishara $\mathrm{H}$ et al also found a considerable delay in the diagnosis of TB among pregnant women. ${ }^{18}$ This and previous studies attributed the diagnostic delays to be on an average 4-26 weeks. ${ }^{19,20}$

The presentation of TB during pregnancy is confounded due to overlapping clinical presentations of TB with normal pregnancy-related conditions, especially in cases of extra pulmonary TB. Compared to non-pregnant women, pregnant women were more likely to be diagnosed with TB via routine screening (as part of prenatal care).

Another reason for this delay is that women seek health services and prenatal care at a late stage of their pregnancy. In HIV infected people, the difficulty in diagnosis is even greater since the weak immune reaction makes early TB symptoms unclear. Thus, most of the studies conducted on interaction of $\mathrm{Tb}$ and pregnancy recommend the integration of $\mathrm{TB}$ screening in prenatal care procedures for high risk groups. ${ }^{21}$
Tuberculosis has been recognized as a maternal and child health issue, as an important cause of morbidity and mortality in pregnancy for nearly a century. A comparison can be made to efforts in addressing HIV infection in pregnancy and the prevention of mother-tochild transmission. Compared to maternal HIV diagnosis, the same attention should be given to maternal TB.

Zenner et al mentioned that compared to risk outside of pregnancy, TB risk was not significantly increased during pregnancy (IRR 1.29, CI 0.82-2.03), but significantly increased post-partum (IRR 1.95, CI 1.24-3.07), adjusting for age, region and deprivation. This raised post-partum TB risk is very likely pregnancy-related, but presents post-partum due to administrative, diagnostic or immunological delays. Combined pregnancy and postpartum TB rates (15.4 per 100,000 person years, PY) were significantly higher than rates outside of pregnancy (9.1 per 100,000 PY, p=0.02).

The significantly increased risk in the 6-month postpartum period may reflect a delay between pregnancy and risk increase for TB. Likely explanations fall into three categories administrative, immunological, and medical. It is also likely that immunological changes gradually increase TB susceptibility during pregnancy, as the expression of T-regulatory cells is partially estrogen dependent and increases throughout the pregnancy. ${ }^{22}$ After delivery, these changes gradually normalize again.

Diagnostic delays occur despite the high level of access to health care enjoyed by pregnant women. Late presentations $^{19}$; an ambiguity of symptoms, frequently mimicking of physiological pregnancy changes ; low index of suspicion amongst medical fraternity and a conservative approach to investigations (e.g. X-rays) have been blamed for these delays. ${ }^{23}$ Delays in treatment initiation will lead to poorer outcomes for mother and fetus.

The WHO Guidelines for Treatment of Tuberculosis provide recommendations for TB care and recommend the integration of $\mathrm{TB}$ care within both prenatal care procedures and the Preventing Mother to Child Transmission of HIV Program (PMTCT) in order to utilise existing health resources and systems to improve accessibility and effectiveness of TB care for pregnant women and prevent the mother to child transmission of TB. ${ }^{24}$

\section{Advantages of screening for $\mathrm{Tb}$ in $\mathrm{ANC}$}

Pregnancy represents an opportunity to address TB in India and other resource-poor countries, where institutional, educational, economic, and cultural barriers prevent many women from accessing healthcare during much of their lives. Perinatal care could be a very good opportunity for TB screening and diagnosis and for following up for TB care, especially for women who have limited access to health services, such as women of 
limited social/economic status, who only approach medical services when pregnant.

Over $75 \%$ of Indian women receive antenatal care, mostly at health care facilities. Untreated pulmonary tuberculosis in pregnant women would be a definite risk for the patient and new-born. It is essential to leverage upon this rare interaction with the healthcare system to optimize preventive health strategies for pregnant women, including TB prevention. Because pregnant women attend multiple antenatal visits, treatment of $\mathrm{Tb}$ during pregnancy also allows close monitoring for potential adverse reactions.

In the context of maternal HIV infection, and also HIVnegative women from high TB burden communities, the WHO prudently recommends TB screening in antenatal clinics. $^{25,26}$

\section{OUR PROPOSAL}

Sputum testing for $\mathrm{Tb}$ to be included for 'cough of any duration' in a pregnant patient as a protocol and implemented by policies and guidelines. The ANC card followed in various states to have an 'entry' for cough of any duration. RNTCP Tb card to make an inclusion of 'pregnancy' as a condition in which 'cough of any duration' to be recorded, thus making a provision for screening of $\mathrm{Tb}$ in pregnancy and better utilizing existing resources, so that a database can be obtained for Tuberculosis in pregnancy. This will not lead to involving any new resource, rather utilise the existing resource to identify new case and increase our case detection rates

\section{Immune changes and their impact on $\mathrm{Tb}$ in pregnancy and puerperium}

Recent knowledge states that women are at increased risk of TB during pregnancy and it is commonly assumed that immunological changes associated with pregnancy present an opportunity for mycobacterial infection or reactivation. ${ }^{28}$ Physiological down-regulation of the cellular immune system is one possible explanation for the higher incidence rates of TB during and immediately after pregnancy.

Pregnancy suppresses the T-helper 1 (Th1) proinflammatory response, which may mask symptoms while increasing susceptibility to new infection and reactivation of tuberculosis. (These effects are seen in other infectious diseases, such as influenza and Mycobacterium leprae, which are more common and severe during pregnancy).

Th1 cytokines (IFN-g and IL-12) are down-regulated. Changes in humoral immunity include increased levels of complement proteins and acute phase reactants, and also increased T-cell dependent immunoglobulin production. $^{28,29}$ Thus pregnant women who are HIV- positive and have LTBI are more likely to progress to active TB disease.

With respect to cellular immunity, increased levels and activity of phagocytes and plasmacytoid dendritic cells have been reported, with down-regulated natural killer (NK) cell cytotoxicity by progesterone-induced blocking factor and interleukin (IL)-10, and also a decrease in interferon gamma (IFN-g) production, indicating a generally suppressed innate cellular response.

After delivery, Th1 suppression reverses similar to immune reconstitution syndrome in HIV patients starting antiretroviral therapy (ART) and symptoms are exacerbated.

A large study recently found that early postpartum women are twice as likely to develop tuberculosis as nonpregnant women. ${ }^{27}$ This and other studies suggest that biologic changes in pregnancy and postpartum influence tuberculosis epidemiology. ${ }^{30}$ Practitioners should be aware of the unpredictable symptomatology of tuberculosis during pregnancy and the varying immune status during different stages of pregnancy and suspect a case of $\mathrm{Tb}$ accordingly.

\section{OUR PROPOSAL}

Since the course of $\mathrm{Tb}$ in pregnancy is unpredictable with confounding symptoms due to immune blunting, the health care provider (at both ends - Tb program related and ante natal care related )to maintain a high degree of suspicion and close follow up for any signs and symptoms of $\mathrm{Tb}$, for a pregnant patient from a high endemic country like India. The follow up to be maintained not only in ANC period but until post-partum period, when the signs and symptoms of $\mathrm{Tb}$ may resurge.

\section{Presentation of $\mathrm{Tb}$ in Pregnancy and post-partum}

Worldwide, an estimated 900 million women have a latent Mycobacterium tuberculosis infection (LTBI), and pregnant women with LTBI are more likely to progress to develop active tuberculous disease than men. India is a high-prevalence nation and there is high exposure to Tuberculosis infection and STCI does not recommend TST and IGRA for diagnosis of active Tuberculosis in India. It has long been observed by obstetricians that pregnancy is associated with a more prevalent onset of active tuberculosis (TB) and also more rapid progression of TB disease compared with the non-pregnant state. ${ }^{31}$

Pregnant patients with active TB often have few of the typical TB symptoms. Between $20 \%$ and $67 \%$ of pregnant patients presenting with pulmonary TB are unaware of their disease and have no significant symptoms. ${ }^{27,32}$

The presentation of tuberculosis in pregnant women is similar to that in non-pregnant women but diagnosis may 
be delayed by the non-specific nature of early symptoms and the frequency of malaise and fatigue in pregnancy. The most common site in pregnancy is pulmonary. Of the 27 pregnancies reported by Good et al with culture positive disease, $74 \%$ had cough, $41 \%$ had weight loss, $30 \%$ had fever, malaise or fatigue, and $19 \%$ had haemoptysis; $20 \%$ were asymptomatic but all had abnormal radiographs.

The disease is twice as likely to reactivate postpartum than at any other time in a woman's life. With nearly 180 million pregnancies occurring annually in moderate/high TB burden settings, and up to $40 \%$ of these women having LTBI, pregnancy is an ideal time to focus on active $\mathrm{Tb}$ case finding. The challenge is identifying pregnant women carrying the infection.

Recent UK data suggest that extra pulmonary TB in pregnancy is as common as pulmonary disease. A history of recent contact with a person with TB or the presence of any of the other risk factors should raise a suspicion of TB. Wilson et al reported that 5-10\% of pregnant women with tuberculosis in their series had extra pulmonary disease, a proportion comparable with nonpregnant women of the same age and ethnic group. A high index of suspicion needs to be maintained. ${ }^{33-36}$

The diagnosis of pulmonary tuberculosis is also complicated by the fact that women with tuberculosis associated with pregnancy are more likely to postpone having chest radiography and that investigation of sputum smear negative tuberculosis is more difficult. Bishara $\mathrm{H}$ et al evaluated a case series at Israel's Northern Health District from 2002-2012.He observed that reluctance to perform a chest X-ray due to fear of harming fetus was a main reason for diagnosis delay in all four pulmonary cases in his case series. ${ }^{18}$
Table 1: New sputum positive (NSP) case detection and cure rates (NMMC hospitals data).

\begin{tabular}{|llll|}
\hline Year & $\begin{array}{l}\text { Suspect } \\
\text { examined Per } \\
\text { Lac }\end{array}$ & $\begin{array}{l}\text { NSP case } \\
\text { detection } \\
\text { rate per } \\
\text { lac }\end{array}$ & $\begin{array}{l}\text { Cure rate } \\
\%\end{array}$ \\
\hline 2010 & 308 & 78 & 86 \\
\hline 2011 & 343 & 84 & 85 \\
\hline 2012 & 248 & 57.25 & 82.25 \\
\hline 2013 & 260 & 59 & 80 \\
\hline 2014 & 280 & 56 & 83 \\
\hline 2015 & 273 & 60 & 84 \\
\hline
\end{tabular}

*Data from NMMC records

\section{OUR PROPOSAL}

In the clinical suspicion of $\mathrm{Tb}$ is seen in a pregnant patient, with a sputum negative result, a decision to perform a timely chest x-ray should not be delayed. The decision is of the clinician on the basis of probability of exposure, past history, and clinical picture. Suitable shielding will limit fetal radiation exposure to less than 0.3 mrads and should not harm the fetus. The perception of teratogenic risk is higher than the actual risk. ${ }^{38}$

\section{The need for timely diagnosis of $\mathrm{Tb}$ in pregnancy}

TB in pregnant women is often an indolent disease with non-specific symptoms at presentation. Diagnosing tuberculosis (TB) in pregnancy can be difficult because of the vague, non-specific nature of the symptoms. Fatigue breathlessness on exertion, malaise, sweating and tired, all characteristic of TB, can also be due to pregnancy. Most physicians are reluctant to order a chest $\mathrm{x}$-ray for fear of harming the fetus. Our goal must be to discover dormant infection and asymptomatic disease and to lower the incidence of congenital or infantile TB.

Table 2: Multi drug resistant (MDR) and total drug resistant (XDR) data (NMMC hospitals data).

\begin{tabular}{|c|c|c|c|c|c|c|}
\hline \multirow[t]{2}{*}{ Year } & \multicolumn{3}{|l|}{ MDR Tb } & \multicolumn{3}{|l|}{ XDR Tb } \\
\hline & Suspects & Diagnosis & Put on treatment & Suspects & Diagnosis & Put on treatment \\
\hline 2010 & 112 & 50 & 36 & $\mathrm{NA}$ & NA & NA \\
\hline 2011 & 377 & 108 & 84 & NA & NA & NA \\
\hline 2012 & 747 & 121 & 102 & & 20 & 7 \\
\hline 2013 & 876 & 99 & 91 & & 15 & 8 \\
\hline 2014 & 857 & 99 & 100 & & 64 & 37 \\
\hline 2015 & 748 & 95 & 77 & & 29 & 20 \\
\hline
\end{tabular}

*Data from NMMC records

Diagnosis of active TB in pregnant patients is similar to that in other patients, with the difference that a significant percentage of pregnant patients with TB will have minimal symptoms and no awareness of their disease.
Practitioners and obstetricians do not often suspect the presence of active TB on clinical grounds. 
Various reports have given multiple perinatal outcomes like increased abortion rates, high levels of preeclampsia, and increased levels of difficult labour requiring intervention in some studies, but overall good fetal outcome in others. ${ }^{39}$ In the last report from the Confidential Enquiry into Maternal and Child Health (CEMACH) there were three indirect maternal deaths due to tuberculous infection. These cases were diagnosed late, possibly because of a lack of awareness of TB in pregnancy among the obstetric and midwifery staff. ${ }^{40}$

In those with a late diagnosis, obstetric morbidity is increased fourfold and preterm labour ninefold. ${ }^{41} \mathrm{~A}$ higher frequency of abortion, toxaemia, and intrapartum complications were reported in another series. A review was made of the records of 27 patients admitted to National Jewish Hospital who developed or experienced a reactivation of pulmonary tuberculosis during pregnancy or the first 12 month post-partum. ${ }^{33,39}$

More recent case control studies have shown that the outcome depends on whether the tuberculosis is pulmonary or extra pulmonary, and also whether it is diagnosed later in pregnancy. ${ }^{48}$

The incidence of prematurity, small for gestational age babies, and low birth weight were more pronounced in cases with late diagnosis, incomplete or irregular drug treatment, and in those with advanced pulmonary lesions, thus implying the importance of timely diagnosis and strict follow up until at least 6 weeks following puerperium.

Figuera-Damien et al reported substantially increased neonatal mortality of $18.7 \%$, a relative risk of 14 , with this being mainly the result of late diagnosis and treatment (during the second and third trimesters). The outcome for those diagnosed in early pregnancy was equivalent to that in non-pregnant patients. ${ }^{41}$

The picture in extra pulmonary tuberculosis is mixed. With lymph node disease no adverse effect was found in a study in India, 72 but for other sites such as the spine, abdomen, and meningitis there were adverse effects on the fetus with a lower Apgar score at birth and the proportion with low birth weight increased to $33 \%$ compared with $11 \%$ in controls.

A heightened index of suspicion and awareness of the rapid advances and innovations made in the diagnosis of TB is also essential for better control of this disease.

\section{OUR PROPOSAL}

Not only pulmonary, the possibility of extra pulmonary $\mathrm{Tb}$ should be well borne in mind of the treating obstetric team to avoid adverse maternal and neonatal outcomes.

\section{Risk for congenital Tb}

Timely diagnosis of TB in pregnant women is essential to prevent congenital $\mathrm{TB}$, intrauterine fetal infection during/after delivery and to prevent TB infection of the new-born. In all cases, proper identification and medical treatment of the Tuberculosis in mother is beneficial to the fetus if the treatment is carefully planned and minimizes the risk of congenital anomalies. Therapeutic abortion is not indicated.

Assuquo B et al conducted a case-control study at three hospitals in Birmingham, comparing pregnant women with TB (2004-6; $n=24)$ with healthy pregnant controls $(n=72)$. Data concerning the course of pregnancy, birth weight and prematurity were collected from review of case notes. The result was that pregnant women with TB are at higher risk of LBW infants. This is particularly true of mothers with pulmonary TB. Mean duration of symptoms was 8.3 weeks so $\mathrm{LBW}$ is attributed to late presentation. Indeed patients have mild symptoms that are confused with those of pregnancy. Therefore a high index of suspicion and early referral and diagnosis is recommended. ${ }^{43}$

It is crucial to rule out TB infection in new-borns whose mothers have been diagnosed with $\mathrm{TB}$, and this investigation should be performed at the time of delivery. Diagnosis of neonatal TB may be perplexing, and the diagnostic delay may have irreversible consequences for the infant. Symptoms of neonatal TB are non-specific; many viral and bacterial infections have similar presentations. Signs and symptoms may include respiratory distress, fever, heptoslenomegaly, lethargy, lymphadenopathy, and irritability. Other signs of TB in neonates include abdominal distension, ear discharge, and skin lesions. An abnormal chest radiograph is not a consistent finding, and a positive PPD test result is even less common.

The initial assessment should include a careful medical evaluation and chest X-rays (the tuberculin skin test is usually negative in new-born infants). Microscopic examination of the placenta to identify any granulomas and taking a TB culture are strongly recommended. Gastric aspiration and cerebrospinal fluid should be microscopically examined and cultured for TB if there is a high clinical suspicion of active TB in the newborn. When there is no clinical evidence of active TB, prophylactic treatment with isoniazid should be considered.

In a prospective series from India, women with disseminated tubercular involvement had higher rates of antenatal hospitalisation, produced infants with low Apgar scores $(\leqslant 6)$ soon after birth, and with low birth weight. $^{44}$ Anemia and congenital anomalies are significantly more common in tuberculosis. Anemia was explained by suppressive influence on the appetite and liver function of pregnant women by specific baciliar 
toxicosis, antituberculous drugs and insufficient resorption of nutrition ingredients (especially iron).

Congenital TB in neonates has morbidity and mortality approaching $50 \%$. We can predict the child to be infected in the uterus if the mother has had miliary TB, TB of the placenta or uterus, or has advanced HIV. ${ }^{46,47}$

\section{OUR PROPOSAL}

Physicians must be aware of the possibility of active TB among pregnant women with risk factors, the reactivation of $\mathrm{Tb}$ in the post-partum period, acknowledge the diagnostic delay and set up a protocol to rule out TB infection of the new-born at delivery by the above mentioned methods.

\section{Need for multidisciplinary approach}

Effective management of TB during pregnancy and the postpartum period requires input from all physicians involved in the care of the mother and fetus i.e. a multidisciplinary approach including an obstetrician, paediatrician, TB specialist, and public health physician. ${ }^{18} \mathrm{~A}$ coherent system of cooperation between the hospital and community services and between paediatricians and adult physicians is vital to find the index adult case to break the chain of contagion as well as to offer prophylactic therapy to the children at risk.

A review of TB diagnoses in Rhode Island, USA from 1987 to 1991 found that pregnant women with TB were most likely to be identified through routine screening and to be asymptomatic. ${ }^{36}$ The same study shows that between $20 \%$ and $67 \%$ of pregnant patients presenting with pulmonary TB are unaware of their disease and have no significant symptoms.

\section{FUTURE RESEARCH PROPOSALS}

There is an urgent need for data and research in $\mathrm{Tb}$ in pregnant and postpartum women in these areas:

- Epidemiology of active tuberculosis

- Immunology and pathogenesis of tuberculosis complicating pregnancy

- Cost-efficacy of screening methods for active tuberculosis and latent tuberculosis

- Epidemiology and management of active tuberculosis and latent tuberculosis in infants born to mothers with tuberculosis.

Women especially pregnant and postpartum women have been underrepresented in clinical trials.

We propose a management algorithm for early detection and management of $\mathrm{Tb}$ in pregnancy and puerperium.

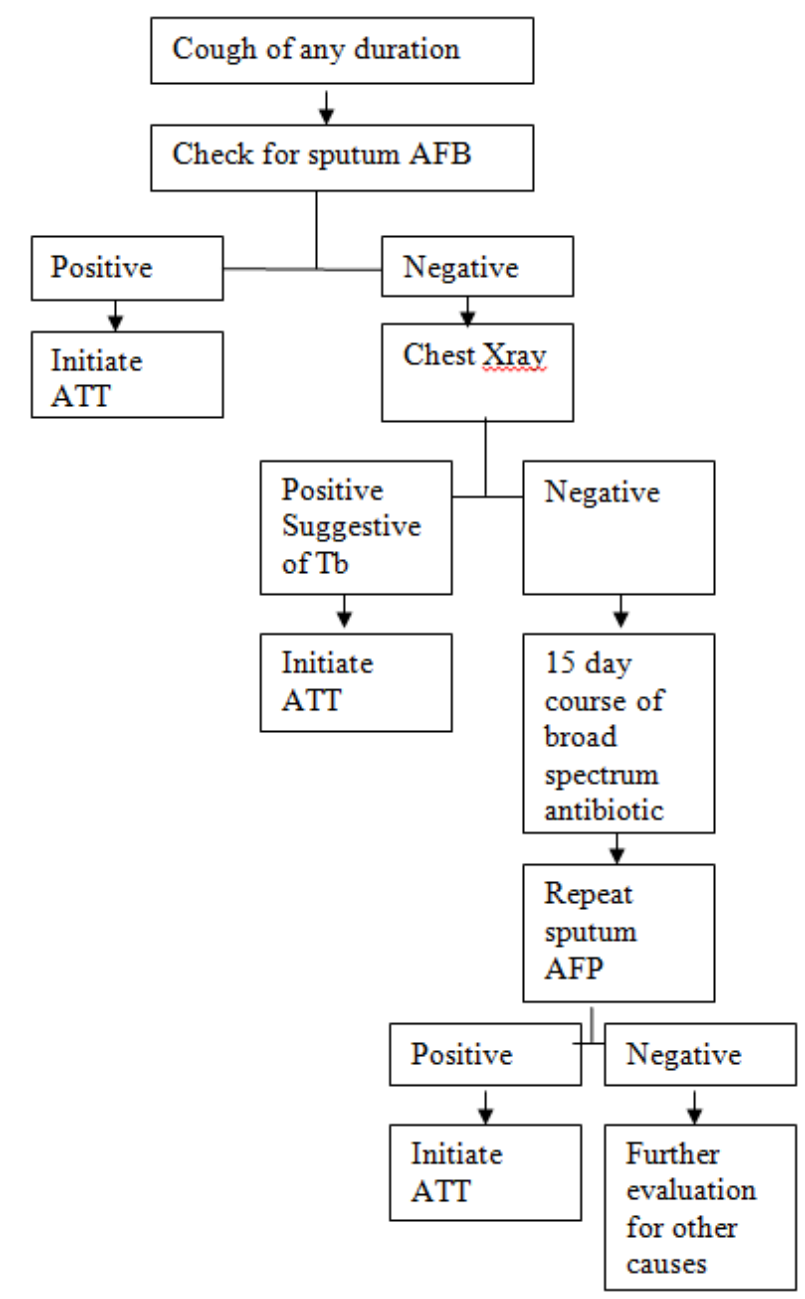

Figure 1: Algorithm proposed for early detection and management of $\mathrm{Tb}$ in pregnancy and puerperium.

\section{CONCLUSION}

Achievement of SDG goal by 2035 requires expanding the scope and reach of interventions for TB care and prevention, with a focus on high-impact, integrated and patient-centered approaches. Our recommendation active TB case screening for pregnant women will utilize available health system resources, especially the antenatal care programs and the existing $\mathrm{Tb}$ programs, and must include well-managed, nation-wide method maintenance of treatment record in order to ensure patients' compliance to TB treatment. This will lead us to identifying more new cases of $\mathrm{Tb}$ by utilizing the existing resources better, optimisation of resource utilisation, thus eliciting full benefits of the program.

Raising doctors' awareness on TB is fundamental. When visiting women with vague symptoms such as low grade fever, malaise and anorexia, doctors should suspect TB and investigate the woman's history and prescribe TB tests in order not to delay diagnosis and to avoid severe consequences. 
Information on individual/family history of TB infection, BCG vaccination, and past treatment, for example, are hardly collected in ANC protocols. These will be of value for correlating cough of any duration. Collecting such information from patients during the first visit and giving this step high priority could help to improve diagnosis and treatment of tuberculosis during pregnancy and improve maternal outcome.

Improvement in TB care for pregnant women will contribute significantly to achieving the Sustainable Development Goal target of ending TB by 2030 and our recommendation of active screening in pregnant females is a unique setting as reinstated by principles of SDG2030.

\section{Funding: No funding sources}

Conflict of interest: None declared

Ethical approval: Not required

\section{REFERENCES}

1. Bates M, Ahmed Y, Kapata N. Perspectives on tuberculosis in pregnancy. International Journal of Infectious Diseases. 2015;32:124-7.

2. Gupta A, Nayak U, Ram M. Postpartum tuberculosis incidence mortality among HIV-infected women their infants in Pune, India, 2002-2005. Clin Infect Dis. 2007;45:241-9..

3. Adhikari M, Pillay T, Pillay DG. Tuberculosis in the newborn: an emerging disease. Pediatr Infect Dis J. 1997; 16:1108-12.

4. Pillay T, Sturm AW, Khan M. Vertical transmission of Mycobacterium tuberculosis in KwaZulu Natal: impact of HIV-1 co-infection. Int J Tuberc Lung Dis. 2004;8:59-69.

5. Khan MS, Dar O, Sismanidis C. Improvement of tuberculosis case detection and reduction of discrepancies between men and women by simple sputum-submission instructions: a pragmatic randomised controlled trial. Lancet. 2007;369:195560.

6. Thorson A, Hoa NP, Long NH. Do women with tuberculosis have a lower likelihood of getting diagnosed? J Clin Epidemiology. 2004;57:398-402.

7. Becerra MC, Pachao-Torreblanca IF, Bayona J. tuberculosis case detection by screening household contacts. Public Health Rep. 2005;120:271-7.

8. "Global tuberculosis control: a short update to the 2009 report," Tech. Rep., World Health Organization, Geneva, Switzerland, (WHO/HTM/TB/2009.426), 2009.

9. 2010/2011 tuberculosis global fact; World Health Organisation. www.who.int/tb/data, Nov. 2010.

10. Connolly M, Nun P. Women and tuberculosis. World Health Stat Q. 1996;49:115-9.

11. Mathad JS, Gupta A. Tuberculosis in pregnant and postpartum women: epidemiology, management, and research gaps. Clin Infect Dis. 2012;55:1532-49.
12. Ormerod P. Tuberculosis in pregnancy and the puerperium. Thorax. 2001;56:494-9.

13. World Health Organisation. World health statistics 2010. Geneva, Switzerland: World Health Organization; 2010.

14. Kothari A, Mahadevan N, Girling J. Tuberculosis and pregnancy: results of a study in a high prevalence area in London. Eur J Obstet Gynecol Reprod Biol. 2006;126:48-55.

15. World Health Organization. Global Tuberculosis Report 2014.

16. Mathad JS, Gupta A. Tuberculosis in pregnant and postpartum women: epidemiology, management, and research gaps. Clin Infect Dis. 2012;55:1532-49.

17. Hoffmann CJ, Variava E, Rakgokong M, Masonoke $\mathrm{K}$, van der Watt M, Chaisson RE, Martinson NA. High prevalence of pulmonary tuberculosis but low sensitivity of symptom screening among HIVinfected pregnant women in South Africa. PLoS One. 2013;8:e62211.

18. Bishara H, Goldstein, Hakim M, Vinitsky O, Shechter-Amram D, Weiler-Ravell D. Tuberculosis during Pregnancy in Northern Israel, 2002-2012: Epidemiology and Clinical Practices. The Israel Medical Association Journal. IMAJ 2015;17(6):34650.

19. Llewelyn M, Cropley I, Wilkinson RJ, Davidson RN. Tuberculosis diagnosed during pregnancy: a prospective study from London. Thorax. 2000;55:129-32.

20. Kothari A, Mahadevan N, Girling J. Tuberculosis and pregnancy: results of a study in a high prevalence area in London. Eur J Obstet Gynecol Reprod Biol 2006;126:48-55.

21. Sheriff FG, Manji KP, Manji MP, Chagani MM, Mpembeni RM, Jusabani AM, et al. Latent tuberculosis among pregnant mothers in a resource poor setting in Northern Tanzania: a cross-sectional study. BMC Infect Dis. 2010;10:52.

22. Lin XG, Zhou Q, Wang L, Gao Y, Zhang WN, Luo ZL, Chen BC, Chen ZH, Chang S. Pregnancy estrogen drives the changes of T-lymphocyte subsets and cytokines and prolongs the survival of $\mathrm{H}-\mathrm{Y}$ skin graft in murine model. Chin Med J (Engl). 2010;123:2593-9.

23. Centers for Disease Control and Prevention. Tuberculosis among pregnant women: New York City, 1985-1992. 1993;42:605-11.

24. World health Organization. Treatment of Tuberculosis: Guidelines for national programmesFourth Edition. Geneva, Switzerland: World Health Organization; 2009.

25. World Health Organization. WHO policy on collaborative TB/HIV activities: guidelines for national programmes and other stakeholders. Geneva: World Health Organization; 2012.

26. Turnbull ER, Kancheya NG, Harris JB, Topp SM, Henostroza G, Reid SE. A model of tuberculosis screening for pregnant women in resource-limited 
settings using Xpert MTB/RIF. J Pregnancy. 2012;2012:565049.

27. Zenner D, Kruijshaar ME, Andrews N, Abubakar I. Risk of tuberculosis in pregnancy: a national, primary care-based cohort and self-controlled case series study. Am J Respir Crit Care Med. 2012;185:779-84.

28. Kraus TA, Engel SM, Sperling RS, Kellerman L, Lo $\mathrm{Y}$, Wallenstein S, et al. Characterizing the pregnancy immune phenotype: results of the viral immunity and pregnancy (VIP) study. J Clin Immunol. 2012;32:300-11.

29. Singh N, Perfect JR. Immune reconstitution syndrome and exacerbation of infections after pregnancy. Clin Infect Dis. 2007;45:1192-9.

30. Espinal MA, Reingold AL, Lavandera M. Effect of pregnancy on the risk of developing active tuberculosis. J Infect Dis. 1996;173:488-91.

31. Stewart DA. Pregnancy and tuberculosis: Part 2 The treatment of tuberculosis complicated by pregnancy. Can Med Assoc J. 1922;12:103-6.

32. Vallejo JG, Starke JR. Tuberculosis and pregnancy. Clin Chest Med. 1992;13:693-707.

33. Good JT, Iseman MD, Davidson PT. Tuberculosis in association with pregnancy. Am J Obstet Gynecol. 1981;140:492-8.

34. Doveren RFC, Block R. Tuberculosis and pregnancy: a provincial study (1990-96). Neth J Med 1998;52:100-6.

35. Hamadeh MA, Glassroth J. Tuberculosis and pregnancy. Chest. 1992;101:1114-20.

36. Carter EJ, Mates S. Tuberculosis during pregnancy. The Rhode Island experience, 1987-1991. Chest. 1994;106:1466-70.

37. Medchill MT, Gillum M. Diagnosis and management of tuberculosis during pregnancy. Obstet Gynecol Surv. 1989;44:81-4.

38. Ratnapalan S, Bona N, Chandra K, Koren G. Physicians' Perceptions of Teratogenic Risk Associated with Radiography and CT During Early Pregnancy. AJR Am J Roentgenol. 2004;182:11.
39. Bjerkedal T, Bahna SL, Lehmann EH. Course and outcome of pregnancy in women with pulmonary tuberculosis. Scand J Respir Dis. 1975;56:245-50.

40. Lewis G. The Confidential Enquiry into Maternal and Child Health. Saving Mothers' Lives: Reviewing Maternal Deaths to Make Motherhood Safer 20032005. The Seventh Report on Confidential Enquiries into Maternal Deaths in the United Kingdom. London: CEMACH; 2007.

41. Figueroa-Damien R, Arredondo-Garcia JL. Pregnancy and tuberculosis: influence of treatment on perinatal outcome. Am J Perinatol. 1998;15:3036.

42. Jana N, Vasishta K, Jindal SK. Perinatal outcome in pregnancies complicated by pulmonary tuberculosis. Int J Gynecol Obstet. 1994;44:119-24.

43. Asuquo B, Vellore AD, Walters G, Manney S, Mignini L, Kunst H. A case-control study of the risk of adverse perinatal outcomes due to tuberculosis during pregnancy. J Obstet Gynaecol. 2012;32(7):635-8.

44. Pranevicius A, Radzeviciūte V, Bimba P, Praneviciūte L. Course of pregnancy, delivery and newborn status in case of maternal tuberculosis. Medicina (Kaunas). 2003;39(4):399-402.

45. Chaulk CP, Moore-Rice K, Rizzo R, Chaisson RE. Eleven years of community-based directly observed therapy for tuberculosis. JAMA. 1995;274:945-951.

46. Nemir RL, O'Hare D. Congenital tuberculosis. Am J Dis Child. 1985;139:284-7.

47. Cantwell MF, Shehab ZM, Costello AM. Brief report: congenital tuberculosis. $\mathrm{N}$ Engl J Med. 1994;330:1051-4.

Cite this article as: Jain KP, Chaudhari PB. Need for increased clinical awareness of tuberculosis during pregnancy and puerperium in India. Int $\mathbf{J}$ Reprod Contracept Obstet Gynecol 2016;5:4106-14. 